\title{
134. The Track of a Rotating Body Travelling near the Surface of Water.
}

\author{
By Nobuji NaSU and Toshiaki ShIRaI. \\ Earthquake Research Institute, Tokyo Imperial University. \\ (Comm. by K. SeZAwA, M.I.A., Dec. 13, 1943.)
}

The object of the present study is to investigate the tracks along which a projected body moves near the surface of water. The path varies with the speed and the sense of rotation that is imparted to the projected body. The interest and importance of the motion of such a body lie in the circulation that ought to be set up around the body when it enters the water. In the simple case of the motion of a solid body in an inviscid fluid, such as is treated in ordinary hydrodynamics, it is evident that there is a definite lift acting on the body when a fluid circulates around it.

The present note concerns certain preliminary experiments that were made, in which a solid circular rotating cylinder was projected with various rotations in direction perpendicular to its length and making a small angle with the surface of the water.

The body of the cylinder (Fig. 1) is of oak, both its ends being protected with aluminium guards: the diameter and length of this cylinder is 40 and $60 \mathrm{~mm}$ respectively. By means of the apparatus shown in Fig. 2, rotation of any speed and in any direction can be given to the cylinder. The cylinder thus rotated is made to strike out in any direction by a specially designed arm (Fig. 3) which is driven by an electric motor. By regulating the speed of this motor it is possible to give any desired traversing speed to the cylinder. In our experiments, however, it was made to rise to such an extent that the velocity of translation of the center of the cylinder was nearly equal to its peripheral velocity.

The results of the experiments so far obtained may be summarised as follows :

(1) The cylinder upon being rotated in sense differing from that of a rolling wheel, and then thrown into water in direction making an angle of less than $10^{\circ}$ with the water-surface, jumped out of the water a number of times, recalling the action of ducks and drakes in water. Reproductions of the cinematographs taken are shown in Fig. 4 , and a part of the tracing of the path is shown in Fig. 5.

(2) When the sense of rotation was the same as that of a rolling wheel, the cylinder invariably sank down into the water after travelling a short distance along its surface (Fig. 6). So long as the cylinder kept rotating, its path was curved, as shown in Fig. 7. Finally it slowly sank down by the action of gravity alone.

(3) When no rotatory motion was imparted to the cylinder, its motion became indefinite; in some cases the cylinder jumped out on the water-surface, in others not. Fig. 8 is an example of the case in 


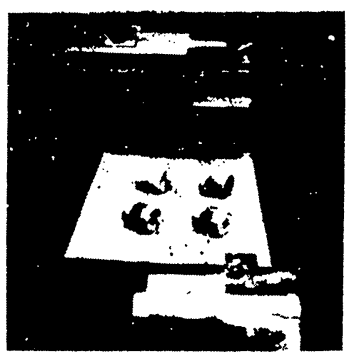

Fig. 1.

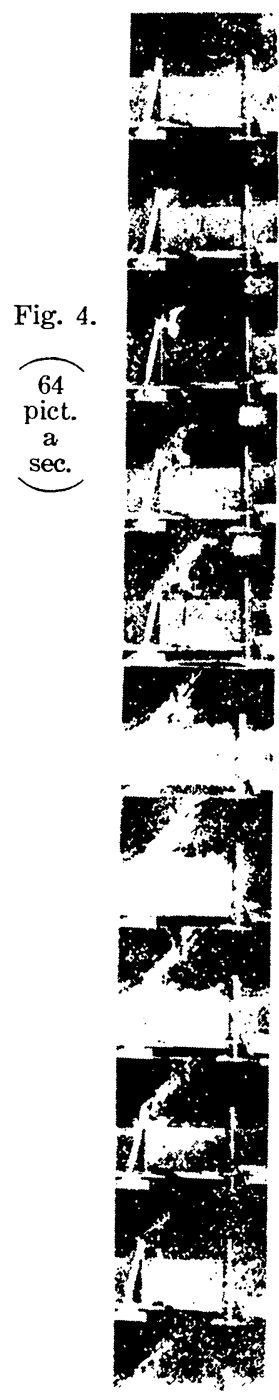

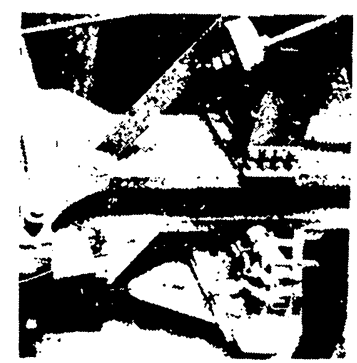

Fig. 2.

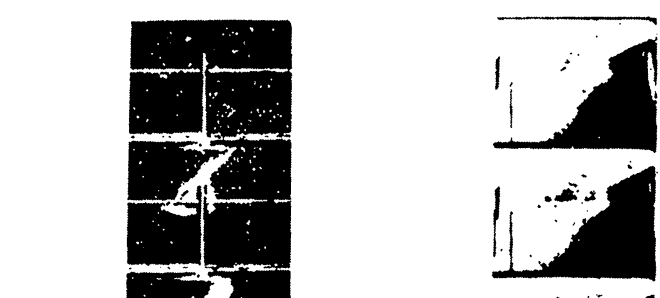

Fig. 6.

16

pict

sec.
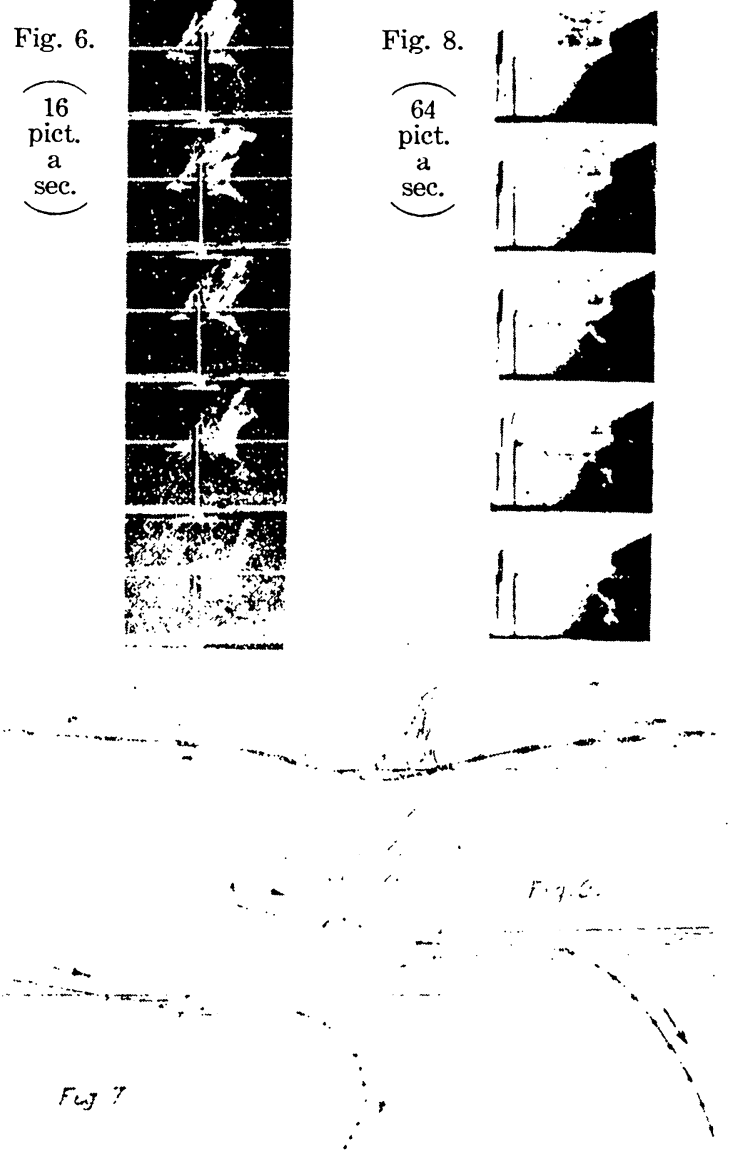
No. 10.] The Track of a Rotating Body Travelling near the Surface of Water. 655

which the cylinder, instead of jumping up, sank down along the path shown in Fig. 9, which should be compared with Fig. 7.

We intend to make further experiments in order to examine the three cases mentioned above in greater detail. 